\title{
Method Development and Validation for the Trace Level Quantification of Genotoxic Impurity Oseltamivir Phosphate Related Compound-A in Oseltamivir Phosphate using LC-MS
}

\author{
PIKKILI VISWANATH ${ }^{1 *}$, DODDIPALLI VENKATA RAMANA REDDY ${ }^{1}$ \\ and NAGARAJU CHAMARTHI ${ }^{2}$
}

\begin{abstract}
'Department of Chemistry, S. G. Govt. Degree and PG College, Piler-517214, Andhra Pradesh, India.
${ }^{2}$ Department of Chemistry, Sri Venkateswara University, Tirupati-517502, Andhra Pradesh, India.

*Corresponding author E-mail: viswa_nath32@yahoo.com
\end{abstract}

http://dx.doi.org/10.13005/ojc/370525

(Received: September 02, 2021; Accepted: October 26, 2021)

ABSTRACT

\begin{abstract}
A selective and sensitive method has been developed for the determination of ethyl-(3S, 4R, 5S)-4-acetamido-5-amino-2-azido-3-(pentan-3-yloxy)cyclohexanecarboxylate (OSPRC-A) by using liquid chromatography coupled with mass spectrometer with single mass analyzer (LC-MS). The method was developed by using column DEVELOSIL ODS-UG-5, $(50 \times 3.0 \mathrm{~mm}, 5.0 \mu \mathrm{m})$ with linearity range of $0.005 \%$ to $0.0151 \%$ which meets to quantification level of $150 \%$ range. The column oven temperature was maintained at $40^{\circ} \mathrm{C}$. The flow rate was set as $1.5 \mathrm{~mL} /$ minute. Injection volume was $10 \mu \mathrm{L}$ and the detection wavelength was $215 \mathrm{~nm}$. The signal to noise ratio values obtained were found to be 4.79 at concentration level of $0.00015 \%$ for the limit of detection (LOD) and 13.46 at concentration level of $0.0005 \%$ for the limit of quantification (LOQ). The $\%$ recovery was found to be in between the range $80.0 \%$ to $101.32 \%$ at LOQ to $150 \%$ level. The result obtained in method precision and intermediate precision are found to be within the specification limit. The percentage RSD for the content of OSPRC-A of method precision was 4.26. The percentage RSD for the content of OSPRC-A for intermediate precision was 4.00. The sample prepared in analytical solution was found to be stable for 24 houre. This method can be used for the identification of impurity, OSPRC-A in Oseltamivir phosphate drug substances in its manufacturing.
\end{abstract}

\section{Keywords: Antiviral drug, Genotoxic impurity, Liquid chromatography, OSPRC-A, Oseltamivir phosphate.}

\section{INTRODUCTION}

Oseltamivir phosphate (OST) is a potent antiviral drug used for the treatment of influenza $A$ and influenza B sold under the brand name Tamiflu. It inhibits the viral enzyme neuraminidase and prevents the virus budding from host cell, infectivity and replication ${ }^{1}$. Oseltamivir phosphate is an ester prodrug metabolized to Oseltamivir carboxylate in the liver by hepatic esterases. The drug half life is 1.8 hours. After oral administration $80 \%$ metabolite shows the therapeutic action for about $30 \mathrm{~min}$ and $20 \%$ of the drug will be present 3-4 $\mathrm{h}$ in plasma. It was found that only $3 \%$ interaction with plasma protein ${ }^{2}$.

This is an Open Access article licensed under a Creative Commons license: Attribution 4.0 International (CC- BY). Published by Oriental Scientific Publishing Company @ 2018 
Tamiflu was the first drug approved in October 1999 by USFDA for the treatment of influenza $A$ and $B$ in adults $^{3}$. OST dosage regimen for adults is $75 \mathrm{mg}$ twice daily for five days, but for prophylactic therapy $75 \mathrm{mg}$ once a day for ten days ${ }^{4}$. OST is described chemically as ethyl (3S, 4R, 5S)-4-acetamido-5amino-3-pentan-3-yloxycylohexene-1-carboxylate phosphate. Its empirical formula is $\mathrm{C}_{16} \mathrm{H}_{31} \mathrm{~N}_{2} \mathrm{O}_{8} \mathrm{P}$ and molecular weight is $410.4 \mathrm{~g} / \mathrm{mol}$. Molecular formula of OST free base is $\mathrm{C}_{16} \mathrm{H}_{28} \mathrm{~N}_{2} \mathrm{O}_{4}$ and molecular weight is $312.4 \mathrm{~g} / \mathrm{mol}^{5}$. The structure of OST and its process related impurities are shown in the Fig.1. Literature survey revealed that oseltamivir phosphate related compound A (OSPRC-A) should be less than 100 ppm in OST when estimated by the LC-MS technique ${ }^{6}$. Different related substances are observed in different synthetic routes and never observed together in a selected synthetic route ${ }^{7}$. As per International Pharmacopoeia the limits of individual impurity in OST is $0.1 \%$ and total impurity should be not more than $0.7 \%$. As a regulatory requirement all the impurities $>0.1 \%$ must be analyzed quantitatively and reported ${ }^{8}$.

The present study is mainly focused on known impurity of OST that is Oseltamivir phosphate related compound $A$ (OSPRC-A) which is produced during the synthesis of Oseltamivir phosphate. The chemical name of OSPRC-A is ethyl (3S, 4R, 5S)4-acetamido-5-amino-2-azido-3-(pentan-3-yloxy) cylohexane-1-carboxylate.Its empirical formula is $\mathrm{C}_{16} \mathrm{H}_{29} \mathrm{~N}_{5} \mathrm{O}_{4}$ and molecular weight is $355.43 \mathrm{~g} / \mathrm{mol}^{9}$. OSPRC-A was found to be mutagenic when present in more than $0.03 \%$. Oseltamivir phosphate passed Ames test when this related substance was not present and reported to be non mutagenic ${ }^{10}$.

\section{Genotoxic impurity}

Genotoxic impurities are the chemical substances which can cause damage to the genetic materials DNA and RNA. Manifestation of genotoxic impurities in APIs and drug products received more attention by pharmaceutical industries and regulatory authorities due to their toxic effects on public health ${ }^{12}$. Young infants, immune compromised patients and severely ill persons are more prone to these genetic effects due to OSPRC- ${ }^{13}$.

\section{LC-MS as detection technique}

LC-MS is a popular and versatile hyphenated technique used for the determination of related substances and impurities at parts per million levels ${ }^{14}$. The literature search disclosed that few methods were developed for estimation of Oseltamivir API alone by HPLC ${ }^{15-16}$, LC/MS/MS ${ }^{17}$, Spectrophotometry ${ }^{18}$, Desorption electro spray ionization mass spectrometry (DESI-MS) ${ }^{19}$.

These factors drawn the attention of the author to develop a method and validate OST and OSPRC-A by highly advanced technique LC-MS to control this related substance in the marketed product.

\section{MATERIALS AND METHODS}

\section{Chemicals and reagents}

OST (as Oseltamivir Phosphate) and its impurity OSPRC-A were supplied by Perfomics Analytical Lab, Hyderabad. HPLC grade methanol, acetonitrile were purchased from Merck, Mumbai, India. HPLC grade water was prepared by using Millipore purification system and used throughout the study. AR grade ethanol, ammonium acetate was procured from SD fine chemicals, Mumbai, India.

\section{Chromatography conditions}

A Waters UPLC TQD mass spectrometer (Waters Corporation, Milford, USA) equipped with PDA detector controlled by Empower software was used for the analysis. We achieved chromatographic separation of OST and Oseltamivir phosphate related compound-A using column DEVELOSIL ODS-UG-55 (C18, $50 \mathrm{~mm} \times 3.0 \mathrm{~mm}$ length, $5.0 \mu$ m diameter) manufactured by the phenomenex. The column oven temperature was maintained at $40^{\circ} \mathrm{C}$. The flow rate was set as $1.5 \mathrm{~mL} /$ minute. Injection volume was $10 \mu \mathrm{L}$ and the detection wavelength was $215 \mathrm{~nm}$. HPLC grade water was used as diluent. Positive ion spray ionization (ESI) probe operated with SIM mode was used for the quantification of OSRPC-A.

\section{Stock, standard and test solution preparation}

Stock solution of OSPRC-A was prepared by weighing accurately about $2 \mathrm{mg}$ of USP OSPRC-A standard into $100 \mathrm{~mL}$ volumetric flask and dissolved in diluent. Working standard solution was obtained by diluting $7.5 \mathrm{~mL}$ of standard stock solution of OSPRC-A into $20 \mathrm{~mL}$ volumetric flask and volume made up to the mark with diluents to get $0.0075 \%$ solution. Standard stock solution of oseltamivir phosphate working standard by weighing accurately 
$50 \mathrm{mg}$ into $5 \mathrm{~mL}$ volumetric flask and dissolved by using solution-A. Test sample solution prepared by weighing $50 \mathrm{mg}$ into $5 \mathrm{~mL}$ volumetric flask by using diluent. Analytical water was used as diluent.

\section{Preparation of buffer solution and mobile phase}

$0.39 \mathrm{~g}$ of accurately weighed ammonium acetate was diluted to $1000 \mathrm{~mL}$ with milli $\mathrm{Q}$ water; its $\mathrm{pH}$ was adjusted to 5.0 with formic acid and filtered through $0.22 \mu$ nylon membrane filter. Mobile phase was prepared by taking a mixture of acetonitrile, water and buffer in the ratio of $3: 6: 1$ $\mathrm{v} / \mathrm{v} / \mathrm{v}$ and filtered through $0.45 \mu$ membrane filter and degassed by ultrasonication.

\section{Optimization of mass spectrometer parameters}

To get the most abundant ion of OSPRC-A, injected the $1 \mathrm{mg} / \mathrm{mL}$ solution through direct infusion method which contain syringe and syringe pump to deliver a regular flow of liquid sample. Initially low sensitivity of ions are observed with flow rate of $10 \mu \mathrm{L} / \mathrm{min}$ at different mass parameters, later increased the flow rate $30 \mu \mathrm{L} / \mathrm{min}$ to get maximum sensitivity of ion by changing the mass parameters in different ways. Final optimized mass parameters are given in Table 1.

Table 1: Optimized mass and source spectrometer parameters

\begin{tabular}{lccccc}
\hline S.No. & \multicolumn{2}{c}{ Mass parameters } & S.No. & Mass parameters \\
\hline 1 & Ionization mode & Electro spray ionization & 7 & Extractor[V] & 3.0 \\
2 & Acquisition mode & Single ion monitoring & 8 & RF lens[V] & 0.3 \\
3 & Polarity mode & Positive & 9 & Source Temp $\left[{ }^{\circ} \mathrm{C}\right]$ & 120 \\
4 & lon & $356.03[\mathrm{M}+\mathrm{H}]^{+}$ & 10 & Desolvation Temp $\left[{ }^{\circ} \mathrm{C}\right]$ & 350 \\
5 & Capillary [KV] & 3.50 & 11 & Cone gas flow $[\mathrm{L} / \mathrm{Hr}]$ & 50 \\
6 & Cone [V] & 25.0 & 12 & Desolvation gas flow [L/Hr] & 950 \\
7 & Split ration & $3: 1$ & & & \\
\hline
\end{tabular}

\section{RESULTS AND DISCUSSION}

Method validation has been performed as per $\mathrm{ICH}$ guidelines. In this study, validation parameters viz; system suitability, specificity, solution stability, LOD and LOQ, accuracy, method precision, linearity and intermediate precision were established to validate the method for the reproducibility of results. System suitability check was done by injecting the six standard solutions and \%RSD was within the limits. Specificity was performed to check the interference at the RT of peak interest with blank, standard and spiking solution and it is seen that there is no peak interference at the retention time of OSPRC-A.

OSPRC-A standard solution was taken and analyzed for solution stability at $5^{\circ} \mathrm{C}$ temperature after 7 and 11 hours. LOD and LOQ were established by considering the concentration of OSPRC-A which would get signal to noise of $3: 1$ and $10: 1$ respectively. Precision was evaluated with six LOQ standard solutions to check the reproducibility of result. Accuracy was established at LOQ, 50\%, 100\% and $150 \%$ interval and recovery found within the specification limit. Method precision was established by spiking the OSPRC-A in OST test sample and $\%$ RSD found satisfactory. Linearity range was conducted for OSPRC-A and the range from LOQ level to $150 \%$ of limit.

\section{Method development}

For the identification of OSPRC-A impurity in Oseltamivir phosphate many trials were done by using different buffers, chemical, solvents and column in isocratic and gradient chromatographic mode by using high performance liquid chromatography (HPLC), but low sensitivity has been achieved in HPLC. Hence, method development trials extended to liquid chromatography coupled with single mass spectrophotometer (LC/MS) to achieve the sensitivity of OSPRC-A at limit of detection level. In addition various additives such as trifluoroacetic acid, formic acid, methanol and acetonitrile have been tested. Number of trials were done by using different buffers, chemicals and solvents like trifluoroacetic acid, formic acid, methanol and acetonitrile and columns which includes $\mathrm{C} 18, \mathrm{C} 8$ and cyano columns to achieve good shape, response and repeatability of results, finally the separation of OSPRC-A and Oseltamivir phosphate achieved in Develosil ODSUG-5 $50 \times 3.0 \mathrm{~mm}$ length, $5.0 \mu \mathrm{m}$ diameter column and mobile phase a mixture of acetonitrile, water and buffer in the ratio of 3:6:1 v/v/v. The flow rate was 1.5 $\mathrm{mL} /$ minute. Determination of OSPRC-A, hampered by inadequately sensitive analytical methods and 
impurity sample volume limitations. Hence, a very sensitive assay for the quantification of OSPRC-A in OST drug substance in manufacturing of OST drug products is highly required without compromising high sensitivity is particularly attractive.

\section{Validation study}

The developed method was validated in terms of system suitability, solution stability, specificity, linearity, accuracy, precision, and robustness, LOQ and LOD. Validation was carried out as per the $\mathrm{ICH}$ guidelines. The method validation was started by injecting $0.01 \%$ solution of OSPRC-A with respect to $5 \mathrm{mg} / \mathrm{mL}$ of OST and their Signal/ Noise ratios determined. Now to determine LOD and $L O Q$ values OSPRC-A concentration was reduced sequentially such that they yield $S / N$ ratios as $3: 1$ and $10: 1$ respectively.

Linearity of OSPRC-A was fixed in the range of LOQ to $150 \%(0.0005 \%-0.0151 \%)$ of the estimated permitted level. Therefore $40 \%, 80 \%$, $100 \%, 120 \%$ and $150 \%$ solutions of OSPRC-A was prepared and injected individually. The calibration curve was plotted between concentration Vs peak area of OSPRC-A. Repeatability was checked by calculating \%RSD of six replicate determinations by injecting six freshly prepared solutions containing $0.01 \%$ of OSPRC-A on the same day. The same experiment was repeated for six different days to find out intermediate precision. The accuracy of the method was determined by spiking known amount of sample at different intervals and spiked with a known amount of OSPRC-A (LOQ to $100 \%$ level).

\section{System suitability}

System suitability performance is a main criterion to know the precision of the instrument. Hence, the system suitability was established by injecting the six replicate injections standard solution and calculated the \%RSD for peak area of Oseltamivir phosphate related compound A. The data was given in Table 2. The \%RSD of OSPRC-A peak obtained from six replicate injections of standard and cumulative \%RSD from six replicate injections with online standard found within the specification limit against pre-defined specification i.e. $\%$ RSD should not be more than $15.0 \%$.

Table 2: System suitability parameters of OSPRC-A

\begin{tabular}{cc}
\hline Injection no. & Area of OSPRC-A \\
\hline 1 & 517685 \\
2 & 526986 \\
3 & 539727 \\
4 & 536846 \\
5 & 529682 \\
6 & 534425 \\
Avg. area & 530891.8 \\
SD & 7964 \\
$\%$ RSD & 1.50 \\
\hline
\end{tabular}

\section{Specificity}

Specificity study established for OSPRC-A by injecting the blank solution and standard solution followed by spiked standard solution to know the interference of any known and unknown impurity at retention time of OSPRC-A. No interference was observed at retention time $(1.160 \mathrm{~min})$ of the OSPRC-A in blank. Retention time of the OSPRC-A were comparable in standard and spiked standard solution. The chromatographic details are shown in Figure 2 and Figure 3.

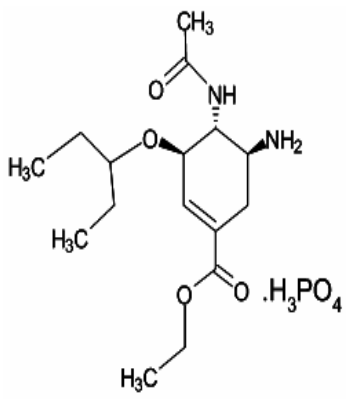

Oseltamivir phosphate (OST)<smiles>CCOC(=O)C1C[C@@H](N)[C@H](NC(C)=O)[C@H](OC(CC)CC)C1N=[N+]=[N-]</smiles>

Oseltamivir Phosphate Related Compound- A
Fig. 1. Chemical structures of Oseltamivir phosphate and OSPRC-A

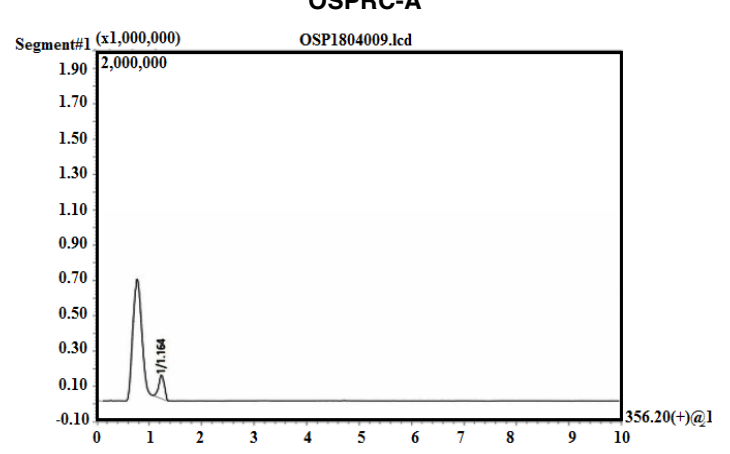

Fig. 2. OSPRC-A standard chromatogram 


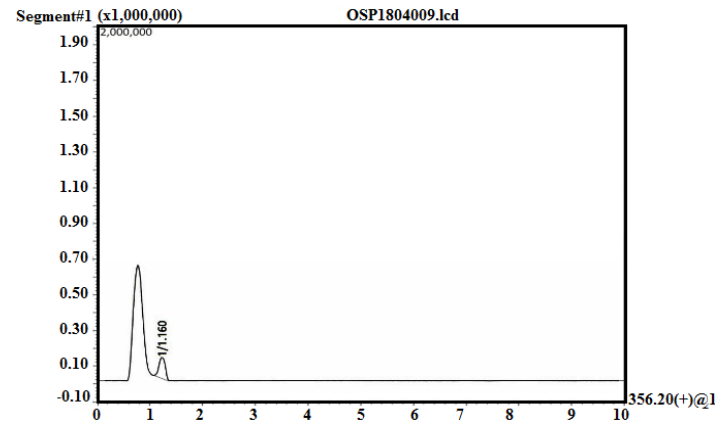

Fig. 3. OST and OSPRC-A spike chromatogram

\section{Solution Stability}

To know the short time shelf life of the analyte in prepared solution, solution stability was established for OSPRC-A in OST sample by injecting the fresh standard and test samples. The solutions were stored at $5^{\circ} \mathrm{C}$ temperature for $7 \mathrm{~h}$ and 11 hours. The variation in the content obtained from solution stability study was within the limit with respect to the defined specification limit $\pm 30 \%$ of the specification limit for genotoxic impurities.

\section{Limit of Detection (LOD)}

Detection limit experiment performed for OSPRC-A to know the lowest detection limit by the method. LOD standard solution was prepared with respect to the test concentration as $0.00015 \%$, so as obtained signal to noise was 4.79 and the chromatogram is shown in Figure 4.

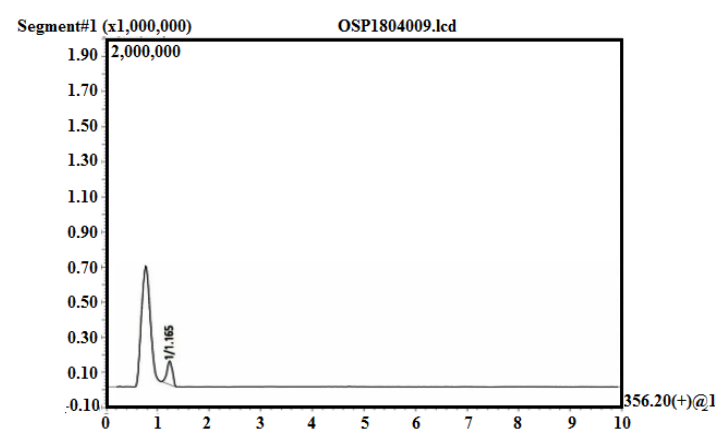

Fig. 4. OSPRC-A LOD chromatogram

\section{Limit of Quantification (LOQ)}

Quantification limit was established for OSPRC-A based on the concentration obtained from LOD, LOQ standard solution was prepared 3 times to LOD concentration $(0.0005 \%)$, so as obtained the $\mathrm{S} / \mathrm{N}$ ratio about 13.46 . The low values of $L O D$ and LOQ indicate an adequate sensitivity of the method.

\section{Precision at LOQ}

To check the closeness of the measurement to the predefined specific limit, precision experiment is performed with LOQ standard solution of OSPRC-A. LOQ standard solution was injected six times and calculated \% RSD. The \% RSD observed for six replicates of LOQ solution was observed to be 1.93 which was well within acceptance criteria, not more than 15.0. The result is summarized in Table 3 and the related chromatogram is shown in Figure 5.

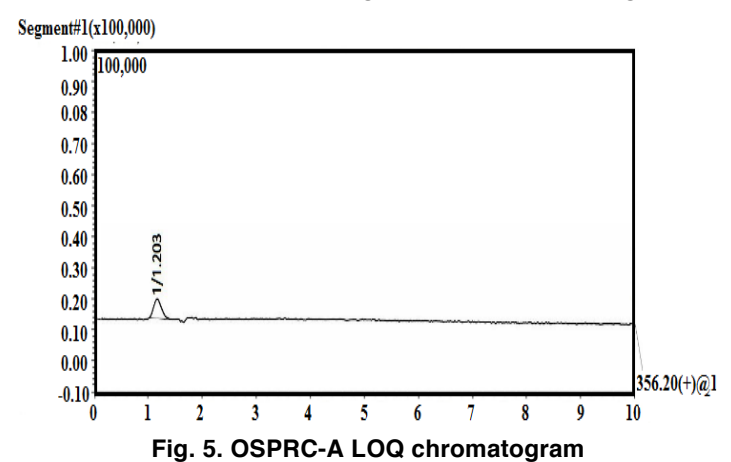

Table 3: Precision of OSPRC-A at LOQ level

\begin{tabular}{cc}
\hline LOQ Standard solution & Area of OSPRC-A \\
\hline Injection-1 & 34871 \\
Injection-2 & 35472 \\
Injection-3 & 35264 \\
Injection-4 & 33647 \\
Injection-5 & 35268 \\
Injection-6 & 34963 \\
Average & 34914 \\
SD & 659 \\
\%RSD & 1.89 \\
\hline
\end{tabular}

\section{Accuracy}

Accuracy study is established to check recovery of analyte when unknown sample spiked in known concentration sample at different levels. So the test sample was analyzed three times (from three individual preparations) for accuracy studies by spiking with OSPRC-A at LOQ, $50 \%, 100 \%$ and $150 \%$ levels and calculated the $\%$ recovery of OSPRC-A in OST. The \%recovery of OSPRC-A was in the range of 80.0 to 101.0 at LOQ, 50\%, 100\% and $150 \%$ level. Accuracy results are summarized in Table 4. It was observed that the method was accurate within the specified range.

Table 4: \%Recovery of OSPRC-A at LOQ, $50 \%$, $100 \%$ and $150 \%$ level

\begin{tabular}{ccc}
\hline Accuracy levels & No. of preparations & \%Recovery of OSPRC-A \\
\hline \multirow{2}{*}{ LOQ } & 1 & 100.00 \\
& 2 & 100.00 \\
$50 \%$ & 3 & 80.00 \\
& 1 & 88.00 \\
$100 \%$ & 2 & 96.00 \\
& 3 & 88.00 \\
$150 \%$ & 1 & 90.10 \\
& 2 & 99.01 \\
& 3 & 97.03 \\
& 1 & 100.66 \\
& 2 & 101.32 \\
& 3 & 100.66 \\
\hline
\end{tabular}




\section{Linearity}

Linearity study was conducted for oseltamivir phosphate related compound $\mathrm{A}$ and the linearity range from LOQ level to $150 \%$ of limit. Correlation coefficient value for OSPRC-A was derived from the linearity graph and it is found to be 0.998. Results are given in Table 5 and the graph is represented in Figure 6.

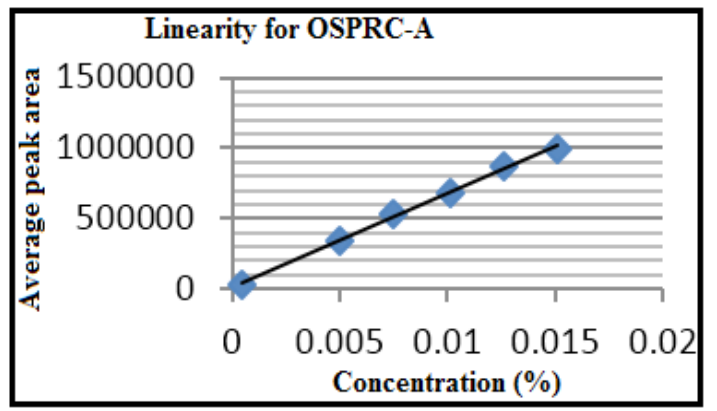

Fig. 6. Linearity graph for OSPRC-A

Table 5: Linearity for OSPRC-A

\begin{tabular}{lcc}
\hline Level & Concentration (\%) & Area of OSPRC-A \\
\hline Level-1 & 0.0005 & 35264 \\
Level-2 & 0.0050 & 344339 \\
Level-3 & 0.0075 & 531982 \\
Level-4 & 0.0101 & 676087 \\
Level-5 & 0.0126 & 864106 \\
Level-6 & 0.0151 & 996329 \\
\hline
\end{tabular}

Correlation coefficient 0.998

\section{Method Precision}

OST test sample was spiked with OSPRC-A at specification level with respect to the test sample concentration for six times and analyzed for the method precision study.

\section{Intermediate precision}

To prove the reproducibility of the method, precision study was done on a different day and with different analyst by using different column with freshly prepared solutions. Variation between the method precision and intermediate precision are given in Table 6 . The result obtained in method precision and intermediate precision are found to be within the specification limit. The percentage RSD for the content of OSPRC-A of method precision was 4.26 (acceptance limits not more than 10.0). The percentage RSD for the content of OSPRC-A for intermediate precision was 4.00 (acceptance limits not more than 10.0).
Table 6: Summary of results for method precision and intermediate precision

\begin{tabular}{lc}
\hline Preparations & Content OSPRC-A (\%) \\
\hline Method precision preparation-1 & 0.0092 \\
Method precision preparation-2 & 0.01 \\
Method precision preparation-3 & 0.0098 \\
Method precision preparation-4 & 0.0091 \\
Method precision preparation-5 & 0.009 \\
Method precision preparation-6 & 0.0093 \\
Intermediate precision preparation-1 & 0.0095 \\
Intermediate precision preparation-2 & 0.0101 \\
Intermediate precision preparation-3 & 0.0101 \\
Intermediate precision preparation-4 & 0.0092 \\
Intermediate precision preparation-5 & 0.0093 \\
Intermediate precision preparation-6 & 0.0091 \\
Average & 0.0095 \\
SD & 0.0004 \\
Cumulative \% RSD & 4.4 \\
\hline
\end{tabular}

CONCLUSION

A simple analytical method was developed and validated for the trace level quantification of genotoxic impurity. OSPRC-A in OST drug substance by using liquid chromatography coupled with mass spectrometer. Full validation was done for the method and established the specificity, linearity, accuracy, precision and ruggedness. The method exhibited an excellent performance in terms of sensitivity and specificity and also found to be simple, precise and cost effective, because the method was developed and validated with single mass analyzer with isocratic mode chromatographic condition. The LOD and LOQ established at low concentration and $S / N$ found to be 4.79 and 13.46 respectively. The sample prepared in analytical solution was found to be stable for 24 hours. Hence this method can be used for the identification of impurity, OSPRC-A in OST drug substances in its manufacturing.

\section{ACKNOWLEDGEMENT}

The authors are thankful to the Perfomics Analytical Lab, Hyderabad for supporting and providing the analytical instruments, chemicals and solvents for development and validation of OSPRC-A by using liquid chromatography coupled with mass spectrometer.

\section{Conflicts of Interest}

The authors declare that there is no conflict regarding publication of this manuscript. 


\section{REFERENCES}

1. Dutkowski, R. Oseltamivir in seasonal influenza cumulative experience in low and high risk patients. J of Antimicrob Chemother., 2010, 65(2), 11-24. doi: 10.1093/jac/dkq012.

2. Aoki, F.Y, Doucette, K. E. Oseltamivir a clinical and pharmacological perspective. Expert Opinion on Pharmacotherapy., 2001, 2(10), 1671-1683. doi: 10.1517/14656566.2.10.1671

3. Gupta, Y., Meenu., M., Mohan, P. The tamiflu fiasco and lessons learnt. Indian J Pharmacology., 2015, 47, 11-16. doi: 10.4103/0253-7613.150308.

4. Dutkowski, R,. Smith., J.R., Davies, B.ESafety and pharmacokinetics of oseltamivir at standard and high dosages. Int J Antimicrob Agent., 2010, 35(5), 461-467. doi: 10.1016/j. ijantimicag.2009.12.023.

5. Drugbank.com, Oseltamivir https:// go.drugbank.com/drugs/DB00198. Accessed, July-3., 2021.

6. The International Pharmacopoeia. 2018. 8th Edition. https://apps.who.int/phint/en/p/docf/ Accesssed, July-3., 2021.

7. Sharma, Y. K., Agarwal, D. D., Bhure, S., Rathore, S. S., Rawat, C., Mukharjee, R Synthesis, isolation and characterization of process related impurities in oseltamivir phosphate. Journal of Chemistry., 2012, 9(1), 113-120. https://doi.org/10.1155/2012/327351.

8. ICH Q3B (R2) Impurities in New Drug Products., 2003.

9. Chemical Book.com, Oseltamivir phosphate related compound $A$. https:// www.chemicalbook.com/ChemicalProduct Property_EN_CB71475965.htm Accessed, July-3., 2021.

10. Pubchem, Oseltamivir, https://pubchem. ncbi.nlm.nih.gov/compound/Oseltamivir Accessed, July-3., 2021.

11. Sagar Kishor Savale. Genotoxicity of drugs introduction, prediction and evaluation. Asian J Biomaterial Res., 2018, 4(6),1-29.

12. Szekely, G., Amores de Sousa, M. C., Gil M., Castelo Ferreira, F., Heggie, W. Genotoxic Impurities in Pharmaceutical Manufacturing Sources, Regulations and Mitigation. Chemical Reviews., 2015, 115, 8182-8229. https://doi.org/10.1021/cr300095f.

13. Reddy, D. Responding to pandemic (H1N1) (2009) influenza the role of oseltamivir. J Antimicrob Chemother., 2010, 65(2), 35-40. doi: $10.1093 / \mathrm{jac} / \mathrm{dkq} 014$.

14. Akshatha, H.S., Gurupadayya, B.M., Application of liquid chromatography coupled with mass spectrometry in the impurity profiling of drug substances and products. Asian J Pharm Clin Res., 2018, 11(5), 3037. https://doi.org/10.22159/ajpcr.2018. v11i5.24212.

15. Narasimhan, B., Abida, K., Srinivas, K., Stability Indicating RP-HPLC Method Development and Validation for Oseltamivir API. Chemical and Pharmaceutical Bulletin., 2008, 56(4), 413-417. doi: 10.1248/cpb.56. 413.

16. Joseph Charles, J., Geneste C., Laborde Kumme E., Gheyouche, R., Boudis, H., Dubost, J. P., Development and validation of a rapid HPLC method for the determination of oseltamivir phosphate in tamiflu and generic versions. J Pharm Biomed Anal., 2007, 44(4), 1008-1013. doi: 10.1016/j.jpba.2007.04.002.

17. Lindegardh, N., Hanpithakpong, W., Wattanagoon, Y., Singhasivanon, P., White, N. J., Day, N.P. J., Development and validation of a liquid chromatographic tandem mass spectrometric method for determination of oseltamivir and its metabolite oseltamivir carboxylate in plasma, saliva and urine. $J$ Chromatogra B., 2007, 859(1), 74-83. doi: 10.1016/j.jchromb.2007.09.018.

18. Omar, M.A., Derayea, S.M., Mostafa, I.M., Selectivity improvement for spectrofluorimetric determination of oseltamivir phosphate in human plasma and in the presence of its degradation product. $J$ of Fluorescence., 2017, 27(4), 1323-1330. DOI: 10.1007/ s10895-017-2066-6.

19. Nyadong, L., Hohenstein, E. G., Johnson, K., Sherrill, C. D., Green, M. D., Fernandez, F. M., Desorption electrospray ionization reactions between host crown ethers and the influenza neuraminidase inhibitor oseltamivir for the rapid screening of tamiflu. The Analyst., 2008, 133(11), 1513. 